\title{
PHOTOIONIZATION OF Ge--DX STATE IN GaAs
}

\author{
R. Piotrzkowski and L.H. Dmowski \\ High Pressure Research Center, Polish Academy of Sciences \\ Sokołowska 29/37, 01-142 Warszawa, Poland
}

\begin{abstract}
We have determined the efficiency of photoionization of $\mathrm{Ge}^{-}-\mathrm{DX}$ state in GaAs as a function of photon energy. The optical ionization energy derived from the fitting is about $1.0 \mathrm{eV}$. It proves a large difference between optical and thermal ionization energies and confirms that for Ge-impurity, the broken-bond model and large lattice relaxation are valid and not the breathing mode with small lattice relaxation, resulting from the calculations presented for Ge-impurity in T.M. Schmidt, A. Fazzio, M.J. Caldas, Mater. Sci. Forum 196/201, 273 (1995).
\end{abstract}

PACS numbers: 63.20.Mt, 71.55.-i, 72.40.+w

\section{Introduction}

Most experimental data for DX-centre related to GaAs:Si and AlGaAs:Si systems can be explained by the broken-bond model with a large lattice relaxation. Ge-impurity, which in contrast to Si substitutes for both host atoms and shows amphoteric behaviour, also creates DX states but with different characteristic than Si. One of the features characteristic for Ge-impurity in GaAs is coexistence of $\mathrm{Ge}^{-}-\mathrm{DX}$ state lying about $110 \mathrm{meV}$ above the bottom of the conduction band, with another strongly localized state the so-called $A_{1}$ or $G^{0}$ state lying about $30 \mathrm{meV}$ below the former [1]. Some theoretical calculations show that for $\mathrm{Ge}^{-}-\mathrm{DX}$ states the broken-bond model usually accepted for DX-centres in III-V compounds is appropriate [2]. However the calculations presented in Ref. [3] indicate that for Ge-impurity the breathing mode with a small lattice relaxation may produce a negative $U$ behaviour, typical of the DX-centres. Determination of the optical ionization energy for $\mathrm{Ge}^{-}-\mathrm{DX}$ state could tell us what is the difference between optical and thermal ionization energies thus confirming either a large or small lattice relaxation for the occupied $\mathrm{Ge}^{-}-\mathrm{DX}$ state.

In this paper we have determined the photoionization cross-section $\sigma_{\text {opt }}$ of $\mathrm{Ge}^{-}-\mathrm{DX}$ state as a function of photon energy. We measured time-dependent photo-Hall effect at $77 \mathrm{~K}$ using hydrostatic pressure to fill the $\mathrm{Ge}^{-}$state. From the photo-Hall measurements we derived $\sigma_{\text {opt }}$ taking into account that some portion of photo-excited electrons from the $\mathrm{Ge}^{-}$is trapped by the $\mathrm{Ge}^{0}$ state which always remains in equilibrium with the conduction band. We compared our results with those for Si-impurity in AlGaAs [4, 5]. 


\section{Experiment}

The GaAs crystal doped with Te was grown by conventional LEC technique. The sample was of the Hall-bar form with dimensions $5 \mathrm{~mm} \times 1 \mathrm{~mm} \times 0.5 \mathrm{~mm}$. The Hall concentration of the measured sample was $8.0 \times 10^{17} \mathrm{~cm}^{-3}$ at $300 \mathrm{~K}$. We used experimental setup designed to study Hall effect and conductivity under hydrostatic pressure at temperatures ranging from $77 \mathrm{~K}$ to ambient with the sample illuminated by monochromatic light. We used a beryllium-copper optical pressure cell with sapphire window, connected by a capillary tube to $1.5 \mathrm{GPa}$ helium-gas pressure generator. A tungsten lamp coupled to a grating monochromator was used as a source of monochromatic radiation. Helmholtz coils wired around the cell supplied a magnetic field. In order to obtain the metastable filling of the resonant $\mathrm{Ge}^{-}-\mathrm{DX}$ level with electrons we proceeded as follows: First, the pressure was raised at room temperature to the value exceeding $1.1 \mathrm{GPa}$ in order to assure a sufficient occupation of $\mathrm{Ge}^{-}-\mathrm{DX}$ state (the occupation of $\mathrm{Ge}^{0}$-state also increases). Next the temperature was lowered to $77 \mathrm{~K}$ (and thus the occupation of $\mathrm{Ge}^{-}-\mathrm{DX}$ state was frozen). Subsequently, the pressure was released at $77 \mathrm{~K}$ (reducing the occupation of $\mathrm{Ge}^{0}$-state which always remains in equilibrium with the conduction band while keeping the occupation of $\mathrm{Ge}^{-}-\mathrm{DX}$ frozen). To find the energy dependence of the photoionization rate we measured the IIall concentration as a function of time under continuous illumination. The initial wa velength of the exciting light was $1.3 \mu \mathrm{m}(0.95 \mathrm{eV})$ and was decreased by $2 \mathrm{~nm}$ between two consecutive measurements.

\section{Results}

The IIall concentration measured as a function of time under continuous illumination with a variable wavelength is shown in Fig. 1. From this dependence we calculate the effective photoionization rate $\alpha$ as

$$
\alpha(h \nu)=I(h \nu) \sigma_{\mathrm{opt}}(h \nu)=-\frac{1}{N_{\mathrm{DX}}(t)} \frac{\mathrm{d} N_{\mathrm{Dx}}(t)}{\mathrm{d} t},
$$

where $N_{\mathrm{DX}}(t)$ is the density of occupied Ge-DX states at the moment $t$ and $I(h \nu)$ is the photon flux. $N_{\mathrm{DX}}(t)$ can be calculated from the measured free electron concentration

$$
N_{\mathrm{DX}}=\frac{N_{\mathrm{D}-\mathrm{Ge}}-\left(n+N_{\mathrm{A}}\right)(1+\xi)}{2+\xi}, \quad \text { where } \quad \xi=g_{0} \exp \left(\frac{E_{\mathrm{F}}-E^{0}}{k T}\right),
$$

$N_{\mathrm{D}-\mathrm{Ge}}, N_{\mathrm{A}}$, and $E^{0}$, correspond to the total Ge-donor concentration, acceptor concentration and the energy of the $\mathrm{Ge}^{0}$ state, respectively. The values of $N_{\mathrm{D}-\mathrm{Ge}}, N_{\mathrm{A}}$, and $E^{0}$, required for the calculation of $\alpha$ and $\sigma_{\text {opt }}$, were derived from the fitting of the ensemble of experimentally measured pressure dependences of electron concentration at $300 \mathrm{~K}$ and $77 \mathrm{~K}$ and temperature dependences at atmospheric and freezing pressures. These values are $N_{\mathrm{D}-\mathrm{Ge}}=3.7 \times 10^{18} \mathrm{~cm}^{-3}, N_{\mathrm{A}}=2.5 \times 10^{18} \mathrm{~cm}^{-3}$, $E^{0}=88 \mathrm{meV}$ at $77 \mathrm{~K}$. The optical ionization cross-section $\sigma_{\mathrm{opt}}$, calculated using. formula (1), is presented in Fig. 2 compared with the results reported by Mooney et al. [5] for AlGaAs:Si. From the fitting of the theoretical expression for $\sigma_{\text {opt }}$ [6] to the results obtained from the experiment we determine the optical ionization energy of Ge-DX state $E_{\text {opt }}$ to be equal to $1.018 \mathrm{eV}$. 

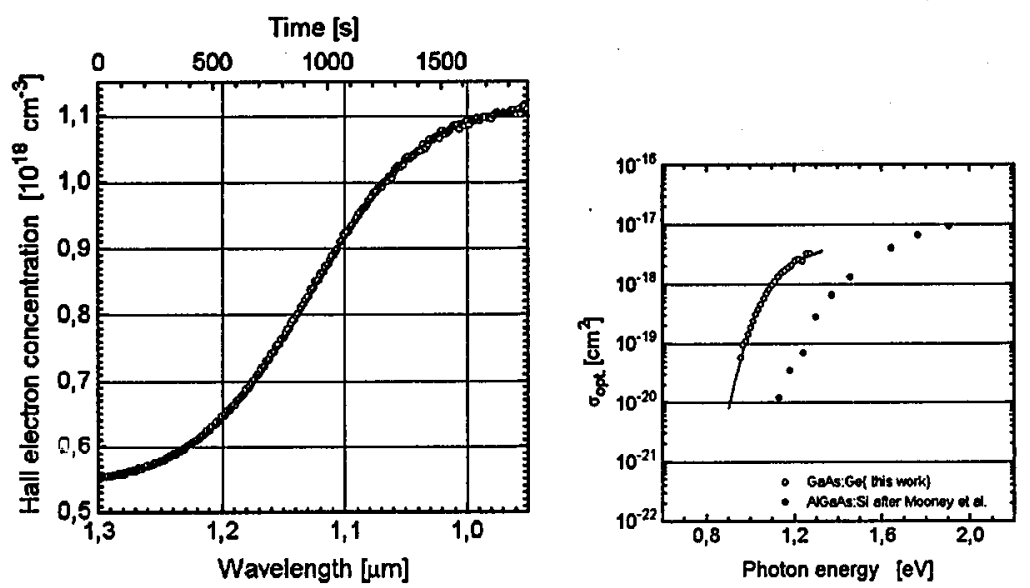

Fig. 1. Hall concentration as a function of time under continuous monochromatic illumination with a variable wavelength. Solid line is the fitting curve taking the optical ionization energy $E_{\mathrm{opt}}=1.018 \mathrm{eV}$ and the values of $N_{\mathrm{D}-\mathrm{Ge}}, N_{\mathrm{A}}$, and $E^{0}$ as mentioned in the text.

Fig. 2. Optical ionization cross-section $\sigma_{\text {opt }}$ of $\mathrm{Ge}^{-}-\mathrm{DX}$ state in GaAs, compared with $\sigma_{o p t}$ for Si-DX state in AlGaAs reported by Mooney et al. [5]. Solid line is the fitting curve taking the optical ionization energy $E_{\text {opt }}=1.018 \mathrm{eV}$.

\section{Conclusions}

We have determined the efficiency of photoionization of $\mathrm{Ge}^{-}-\mathrm{DX}$ state as a function of photon energy. The obtained effective photoionization rate $\alpha$ and the optical ionization cross-section $\sigma_{\text {opt }}$ occur to be very close to those reported for Si-impurity which is well established to be described by the broken-bond model. The high optical ionization energy $E_{\mathrm{opt}}=1.018 \mathrm{eV}$ obtained for $\mathrm{Ge}^{-}-\mathrm{DX}$ state being resonant with the conduction band confirms the large lattice relaxation model for Ge-DX centre in GaAs.

\section{References}

[1] M. Baj, L.H. Dmowski, T. Słupinski, Phys. Rev. Lett. 71, 3529 (1993).

[2] S. Bednarek, J. Adamowski, in: Proc. of ICPS-23, Berlin 1996, Eds. M. Scheffler,

R. Zimmerman, World Scientific, Singapore 1996, p. 2781.

[3] T.M. Schmidt, A. Fazzio, M.J. Caldas, Mater. Sci. Forum 196/201, 273 (1995).

[4] R. Piotrzkowski, E. Litwin-Staszewska, J. Przybytek, Phys. Status Solidi B 198, 205 (1996).

[5] P.M. Mooney, G.A. Northrop, T.N. Morgan, H.G. Grimmeiss, Phys. Rev. B 37, 8298 (1988).

[6] U. Piekara, J.M. Langer, B. Krukowska-Fulde, Solid State Commun. 23, 583 (1977). 